\title{
Professional Life and Sports
}

\author{
Jörg Bahm
}

\subsection{Sports}

In general swimming is beneficial for the development of muscle strength and riding for a good alignment of the axial posture; but many boys want to play football first, which will certainly improve the general health condition. No sport is forbidden or contraindicated, unless the fun factor is completely lost or severe stress pain occurs again and again.

Sport also means social integration!

How this can appear in everyday life can be read in the personal experience reports in Chap. 26.

Even over long periods of time, sports muscle training in patients with nerve damage does not lead to unlimited muscle buildup and the attainment of normality, the unconscious desire of all parents to reverse the initial damage, but it does help to bring general physical fitness together with weight control into health care.

There is also much dynamism in disabled sports; we are currently running a nationwide swimming campaign with the self-help group "Plexuskinder" (www.plexuskinder.de).

\footnotetext{
J. Bahm $(\bowtie)$

Plastic, Hand and Burn Surgery, Section for Plexus Surgery, University Hospital, Aachen, Germany e-mail: jorg.bahm@belgacom.net, jbahm@ukaachen.de
}

\subsection{Occupation}

The choice and practice of a profession imply a long-term perspective, which must take into particular account the continuing physical strain on the upper extremity: I do not recommend heavy, repeated, often one-sided and two-sided physical strain as encountered when one works, for example, as a heating installer. In addition, counselling and classification by the national compensation office can also help here (Chap. 29). Considering the disablement degree, controversies often arise regarding an alleged undervaluation, and especially in the case of a slight degree of movement restriction, the question must be asked whether one wants the "stamp" related to disability or wants to enter adulthood without any mark.

Every one-sided affected person will be able to cope in our working world, which is so rich in aids - this is my first message to parents and children at the first consultation. But of course it is a long process of learning, adaptation, and sometimes even renunciation until then.

The choice of profession and the world of careers are another chapter of probation, where all therapists should, of course, be available for advice.

Prudent courage, taking into account the specific limitations, socialization, and a good acceptance of the body image and limitation, coupled with a healthy self-esteem, are important and universal prerequisites for a balanced adult life in the midst of our society.

The term "disability" should be avoided! 